\title{
Image Retrieval based on the Multiwavelets Texture-Spatial Features
}

\author{
An Zhiyong ${ }^{1, *}$, Li jinjiang $^{1}$, Zhao feng ${ }^{1}$ and Guo jie ${ }^{2}$ \\ ${ }^{1}$ Key Laboratory of Intelligent Information Processing in Universities of Shandong (Shandong Institute of Business and Technology), \\ Yantai, china \\ ${ }^{2}$ Shandong Normal University, Jinan, China
}

Received: 6 Apr. 2013, Revised: 7 Aug. 2013, Accepted: 9 Aug. 2013

Published online: 1 Mar. 2014

\begin{abstract}
A new retrieval algorithm based on the texture-spatial texture using the GHM multiwavelets is presented. In order to describe the important visual information of image, we design the improved multiwavelets quantization map that can depict the important visual information for the multiwavelets sub-bands. Furthermore, the visual spatial histogram of multiwavelets quantization map is used as the texture-spatial features that denote the global texture information of image. At the same time, the local binary pattern is used to describe the local texture-spatial feature for the low frequency multiwavelets sub-band. Finally, the similarity of visual spatial histogram is computed by the spatial-weighted distance. Experiments indicate that this method gives better performance than looseness texturespatial algorithm and MTH algorithm in the natural image retrieval.
\end{abstract}

Keywords: Content-based image retrieval, multiwavelets transform, texture feature

\section{Introduction}

With the rapid growth of digital image, content-based image retrieval (CBIR) become important research area to help people to retrieve useful information.

Wavelet transform is one of effective multiresolution approach to describe the texture feature nowadays. There are many retrieval algorithms using wavelet transform. Han [1] put forward the rotation-invariant and scale-invariant texture retrieval algorithms using the Gabor wavelet for the geometric image database. Quellec [2]proposed the retrieval algorithm using the bi-orthogonal wavelets that yields a good performance in retrieving the medical database. Manesh [3]proposed texture image retrieval algorithm using the new rotated complex wavelet that significantly improves retrieval performance over the traditional approach and retains comparable levels of computational complexity. Shang [4] proposed the multiwavelets histogram method that perform the good retrieval-rate for texture image. Since multiwavelets has the advantage of short support, orthogonally, symmetry and high order vanishing moments simultaneity, multiwavelets histogram method provides a good indexing performance for natural images. However, the multiwavelets histogram method only considers the global distribution and cannot depict local distribution.

Reference [5]introduced the looseness feature based on the integrated multiwavelets quantization map. Liu [6] presented a multi-texton histogram $(\mathrm{MTH})$ method that integrates spatial information and the advantages of co-occurrence matrix by representing the attribute of co-occurrence matrix using histogram. However, the above methods only describe the global spatial information about pixels arrangement and lose the local spatial information.

In order to improve retrieval rate for the natural image, we propose a new retrieval algorithm based on the texture-spatial features. The rest of this paper is organized as follows. In Section 2, we introduce briefly the multiwavelets transform. In Section 3, the multiwavelets texture features and the image retrieval algorithm are presented. In section 4, the procedure to retrieve texture images and the experimental results are presented. In section 5 , we discuss the results and conclusion.

\footnotetext{
*Corresponding author e-mail: azytyut@163.com
} 


\section{Multiwavelets Transform}

Multiwavelets is defined using several wavelets with several scaling functions and has several advantages in comparison with scalar wavelet. Multiwavelets transform not only has good time and frequency localization characteristic, but also has the property of the smooth,tight support, symmetry,orthogonal together. Let $\phi(x)=\left[\varphi_{1}, \varphi_{2}, \cdots, \varphi_{r}\right]^{T}$ be a vector-valued function belonging to $\varphi_{\mathrm{i}} \in L^{2}(R)$. Define, for $j \in Z$

$$
V_{j}=\overline{\operatorname{span}\left\{2^{j / 2} \varphi_{i}\left(2^{j} x-k\right): 1 \leq i \leq r, k \in Z\right\}}
$$

Definition 2.1 [7]: $\phi$ is called a multiscaling function if the spaces defined in (1) satisfy the following properties:

(1) $\cdots \subset V_{0} \subset V_{1} \subset V_{2} \subset \cdots$

(2) $\overline{\cup V_{j}}=L^{2}(R) ; \cap V_{j}=\{0\}$;

(3) $f \in V_{j} \Leftrightarrow f(2 x) \in V_{j+1}$

(4) $f \in V_{j} \Leftrightarrow f(x-k) \in V_{j}, \forall k \in Z$

(5) $\left\{\varphi_{i}\left(2^{i} x-k\right): 1 \leq i \leq r, k \in Z\right\}$ is a Riesz basis for $V_{j}$.

For an assigned MRA of multiplicity $\left\{V_{j}\right\}_{j \in Z}, W_{j}$ can be defined as the complementary space of $V_{j}$ for every $j \in Z$.The $V_{j}+1$ is the direct sum of $V_{j}$ and $W_{j}$, namely $V_{j+1}=V_{j} \oplus W_{j}$.Let $\psi \mathrm{a} \quad$ vector-valued function $\psi(x)=\left[\psi_{1}, \psi_{2}, \cdots, \psi_{r}\right]^{T}, \psi_{i} \in L^{2}, i=1,2, \cdots, r$ ,such that

$$
W_{j}=\overline{\operatorname{span}\left\{2^{j / 2} \psi_{i}\left(2^{j} x-k\right): 1 \leq i \leq r, k \in Z\right\}}
$$

Definition2.2 [7]:The above defined vector-valued function $\psi$ is a semi-orthogonal multiwavelets if, for every $j \in Z$,it satisfies the following conditions $V_{j} \perp W_{j}$, the family $\left\{2^{j / 2} \psi_{i}\left(2^{i} x-k\right): 1 \leq i \leq r, k \in Z\right\}$ is a stable basis for $W_{j}$.

Multiwavelets offer a higher degree of freedom for image processing applications, compared with scalar wavelets. Multiwavelets require the preprocessing that the input signal is to be first vectored. Strela [8]provided two peprocessing of methods: repeated row preprocessing (over sampling) and matrix preprocessing (critical sampling). The repeated row preprocessing can generate more information than the matrix preprocessing. Therefore, we will use repeated row preprocessing as the preprocessing method for multiwavelets transform. Since multiwavelets decomposition produce two low pass subbands and two high pass subbands in each dimension, the organization and statistics of multiwavelets subbands differ from the scalar wavelet case. The multiwavelets have two channels, so there will be two sets of scaling coefficients and two sets of multiwavelets coefficients. Figure 1 shows the decomposed image using the GHM multiwavelets transform for the Lena image. From Figure 1 , we can find that the low pass LL is actually a sub block of subbands (the $L_{1} L_{1}, L_{1} L_{2}, L_{2} L_{1}$ and $L_{2} L_{2}$ subbands).

\begin{tabular}{|l|l|l|l|}
\hline $\mathrm{L}_{1} \mathrm{~L}_{1}$ & $\mathrm{~L}_{1} \mathrm{~L}_{2}$ & $\mathrm{~L}_{1} \mathrm{H}_{1}$ & $\mathrm{~L}_{1} \mathrm{H}_{2}$ \\
\hline $\mathrm{L}_{2} \mathrm{~L}_{1}$ & $\mathrm{~L}_{2} \mathrm{~L}_{2}$ & $\mathrm{~L}_{2} \mathrm{H}_{1}$ & $\mathrm{~L}_{2} \mathrm{H}_{2}$ \\
\hline $\mathrm{H}_{1} \mathrm{~L}_{1}$ & $\mathrm{H}_{1} \mathrm{~L}_{2}$ & $\mathrm{H}_{1} \mathrm{H}_{1}$ & $\mathrm{H}_{1} \mathrm{H}_{2}$ \\
\hline $\mathrm{H}_{2} \mathrm{~L}_{1}$ & $\mathrm{H}_{2} \mathrm{~L}_{2}$ & $\mathrm{H}_{2} \mathrm{H}_{1}$ & $\mathrm{H}_{2} \mathrm{H}_{2}$ \\
\hline
\end{tabular}

(a) Multiwavelets structure

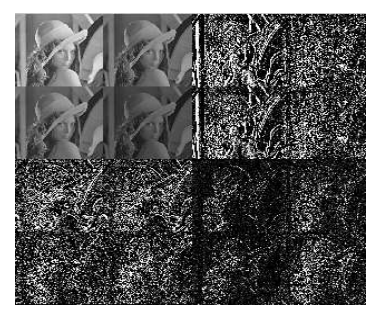

(b) Decomposition image

Figure 1: Multiwavelets decomposition subbands for single level decomposition

\section{Extraction of Multiwavelets Signatures}

\subsection{The Improved Multiwavelets Quantization Map}

In order to get the important texture information, we decompose the retrieval image with the three levels of multiwavelets transform. Therefore, there are ten channels and four sub blocks in each channel. Reference[5] make use of the ten channels to design the integrated multiwavelets quantization map. However, the integrated multiwavelets quantization map has the shortcomings of high complexity and redundant texture information.

Here we will use the nine channels that consist of $\mathrm{LH}_{2}, H L_{2}, H H_{2}, L H_{1}, H L_{1}, H H_{1}, L H_{0}, H L_{0}, H H_{0}$ to make the multiwavelets quantization map because the high frequency sub-bands contain more texture details. Firstly, the magnitudes matrix for every sub-band is computed. The $0 / 1$ quantization is used to deal with the magnitudes matrix of sub block of multiwavelets decomposition. Let $w[i, j]$ be a coefficient of a block matrix and $T_{K}$ is threshold in sub block such that

$$
M(i, j)=\left\{\begin{array}{l}
1|w(i, j)| \geq T_{K} \\
0
\end{array}\right.
$$

In order to make the threshold self-adoptive, we will use the mean of sub block as the threshold. Therefore, a texture point can be defined as a 9-D vector by considering channel values from the same location of all sub bands. These binary data can be transformed as the decimal data. Figure 2 shows the construction for a texture 
point.

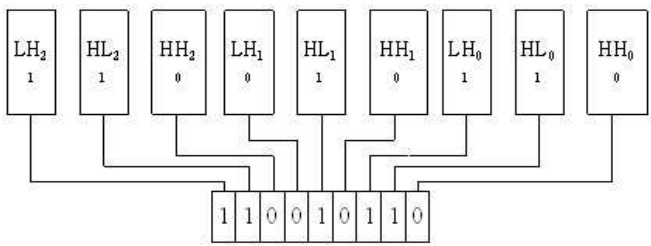

Figure 2: The construction for a texture point

All the texture points can constitute a multiwavelets quantization map that describes the texture information of image. It is noted that the coefficients of multiwavelets quantization map are positive integer that is convenient for the feature extraction. Figure 3 is the multiwavelets visual quantization map for the Lena.

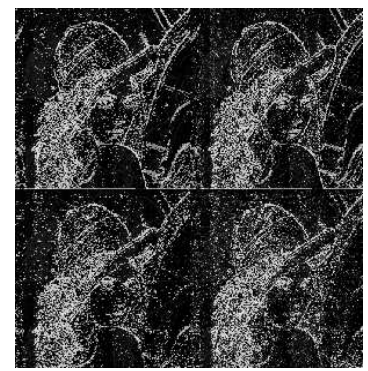

Figure 3: The multiwavelets quantization map

However, the multiwavelets quantization map can not adapt to the human visual perception. Therefore, the four sub-blocks of multiwavelets quantization map should be integrated as a new quantization map. Suppose the four sub-blocks is named as the $S_{1}, S_{2}, S_{3}$ and $S_{4}$. The improved multiwavelets quantization map can be defined as

$$
\operatorname{map}(i, j)=\left(S_{1}(i, j)+S_{2}(i, j)+S_{3}(i, j)+S_{4}(i, j)\right) / 4
$$

The improved multiwavelets quantization map can be seen as a whole standardization map for all sub-bands. Since we only use the nine high frequency sub-bands to make the improved multiwavelets quantization map, it has the lower redundancy than the integrated multiwavelets quantization map [5].

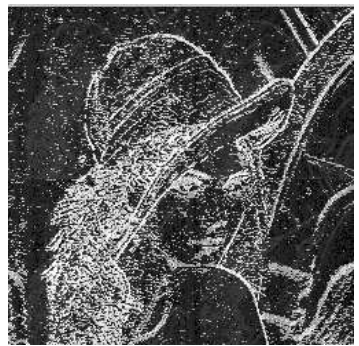

Figure 4: The improved multiwavelets quantization map

\subsection{Global Spatial-histogram Feature}

Let $C_{i}=\left(x_{i}, y_{i}\right)$ be the center of mass of bin and $\left|H_{i}\right|$ the count of pixels in bin i, where $\left(x_{i}, y_{i}\right)$ can be defined as:

$$
\left\{\begin{array}{l}
x_{i}=\frac{1}{\left|H_{i}\right|} \sum_{(x, y) \in H_{i}} x_{i} \\
y_{i}=\frac{1}{\left|H_{i}\right|} \sum_{(x, y) \in H_{i}} y_{i}
\end{array}\right.
$$

Let $\sigma_{i}$ be the standard deviation of bin i from $C_{i}$ and it is defined as

$$
\sigma_{i}=\sqrt{\frac{\sum_{p \in H_{i}} d\left(p, C_{i}\right)^{2}}{\left|H_{i}\right|}}
$$

where $\mathrm{p}$ is the position of a pixel at $(\mathrm{x}, \mathrm{y})$ in multiwavelets quantization map and $d\left(p, C_{i}\right)$ is defined as

$$
d\left(p, C_{i}\right)=\sqrt{\left(x-x_{i}\right)^{2}+\left(y-y_{i}\right)^{2}}
$$

The spatial histogram index for quantization map is $H=\left\{\left(H_{1}, \sigma_{1}\right), \cdots,\left(H_{n}, \sigma_{n}\right)\right\}$, where $\sigma_{i}$ measures the square root of the average squared distance of pixels in bin i from its center. Let $\mathrm{Q}$ denotes queering image and I denotes an image of image database. Then, the similarity metric of image $\mathrm{Q}$ and $\mathrm{I}$ is defined as:

$$
d_{s}(Q, I)=\sum_{i=1}^{N} \min \left(H_{i}^{Q}, H_{i}^{I}\right) \times \frac{\min \left(\sigma_{i}^{Q}, \sigma_{i}^{I}\right)}{\max \left(\sigma_{i}^{Q}, \sigma_{i}^{I}\right)}
$$

The similarity metric is made up of two parts. The first $\operatorname{partmin}\left(H_{i}^{Q}, H_{i}^{I}\right)$ measures the similarity between $H_{i}^{Q}$ and $H_{i}^{I}$. The second $\operatorname{part} \frac{\min \left(\sigma_{i}^{Q}, \sigma_{i}^{I}\right)}{\max \left(\sigma_{i}^{Q}, \sigma_{i}^{I}\right)}$ easures the similarity of the spatial distribution of the pixels of a bin. It is noted that the $d_{s}$ described in formula 8 is rotation invariant. 


\subsection{Local Binary Pattern Feature}

Local Binary Pattern(LBP) is an efficient texture operator which labels the pixels of an image by threshold the neighborhood of each pixel [9]. Here, we can use the LBP to describe the local texture-spatial feature for the multiwavelets low frequency sub-band. Let gc denote the gray value of the center pixel of the local neighborhood and gp denote the gray values of $\mathrm{P}$ equally spaced pixels on a circle of radius $\mathrm{R}$ that form a circularly symmetric neighbor set.The value of the LBP code of a $\operatorname{pixel}\left(x_{c}, y_{c}\right)$ is given by

$$
\mathrm{LBP}_{\mathrm{P}, \mathrm{R}}=\sum_{\mathrm{p}=0}^{\mathrm{p}-1} \mathrm{~s}\left(\mathrm{~g}_{\mathrm{p}}-\mathrm{g}_{\mathrm{c}}\right)
$$

where $s(x)$ can be defined as

$$
s(x)=\left\{\begin{array}{l}
1, \text { if } \geq 0 \\
0, \text { otherwise }
\end{array}\right.
$$

Then, theLBP $P_{P, R}$ histogram can be used to describe the texture feature of low frequency sub-band. Let $\mathrm{Q}$ denotes queering image and I denotes an image of image database, the texture similarity can be calculated by the (11).

$$
d_{L B P}\left(H_{L B P 1}, H_{L B P 2}\right)=1-\frac{\sum_{i=1}^{n} \min \left(h_{L B P 1}^{i}, h_{L B P 2}^{i}\right)}{\min \left(\sum_{i=1}^{n} h_{L B P 1}^{i}, \sum_{i=1}^{n} h_{L B P 2}^{i}\right)}
$$

where $\mathrm{n}$ is the number of bins.

\subsection{The Algorithm Description}

The improved multiwavelets quantization map describes the relation information among the different sub-bands. The spatial histogram describes the global texture information for the quantization map. Thus, the spatial histogram and LBP feature are mutually complementary for the texture features. Then, the similarity between two images can be defined as the distance

$$
D(P, Q)=\omega_{1} d_{s}+\omega_{2} d_{L B P}\left(\omega_{1}, \omega_{2}>0, \omega_{1}+\omega_{2}=1\right)
$$

where $\omega_{1}$ is the weight of spatial histogram and $\omega_{2}$ is the weight of the LBP. The image retrieval algorithm can be described as follows.

STEP1: The original image is decomposed of three levels by the multiwavelets transform.

STEP2: The multiwavelets quantization map is computed for the magnitudes matrix of multiwavelets sub-bands. The spatial histogram index for quantization map is extracted as the global texture information.
STEP3: The local binary pattern can be used to describe the texture feature of low frequency sub-band.

STEP4: The texture similarity of the querying image and other images is computed by Eq.(12).

\section{Performance of the Algorithm}

To evaluate the retrieval efficiency of proposed algorithm, the natural image database (http://Wang. ist.psu.edu/) is used that contains 2300 different images, including bus, horse, flower animals and birds. Here the average retrieval rate is used to appraise the performance for the retrieval algorithm. The average retrieval rate for the query image is measured by counting the number of images from the same category which are found in the top $\mathrm{N}$ matches[10].

The proposed algorithm is compared with the looseness texture-spatial algorithm and MTH algorithm. Here we make the weight $\omega_{1}=\omega_{2}=0.5$.It means that the LBP and the spatial histogram have the equal weight. Figure 5 shows the comparative average retrieval rates for the three algorithms. From Figure 5, it can be seen that the proposed algorithm gives the best performance in the natural image retrieval. The superiority of the proposed method is also observed in all the cases, i.e., when $\mathrm{N}$ is considered as $1,5,10,15,20,25,30(\mathrm{~N}$ is the number of top retrieved images). When all the images in a class are retrieved $(\mathrm{N}=30)$ proposed algorithm is able to produce an improved average retrieval rate of 3.49 .

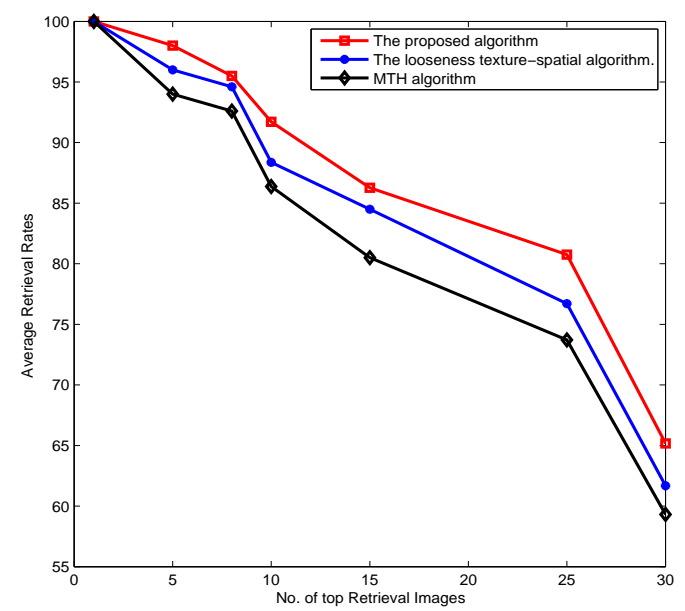

Figure 5: Comparative average retrieval rates

Since MTH algorithm only analyzes the global texture information, the average retrieval rate is lower than the proposed algorithm. Though the looseness texture-spatial algorithm analyzes the sub-bands array for texture, it lost the local texture-spatial information for texture. At the 
same time, the improved multiwavelets quantization map contains the little redundant texture information than that of looseness texture-spatial algorithm. Therefore, the proposed algorithm yields better retrieval accuracy than looseness texture-spatial algorithm using the multiwavelets in the natural image retrieval.

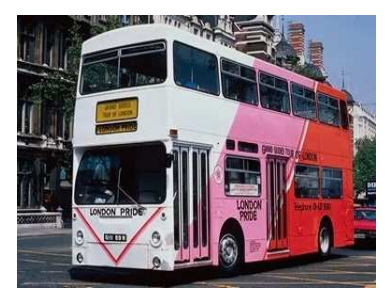

(a) Retrieval image
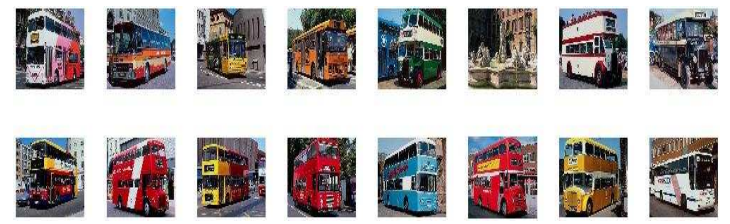

(b) The proposed algorithm
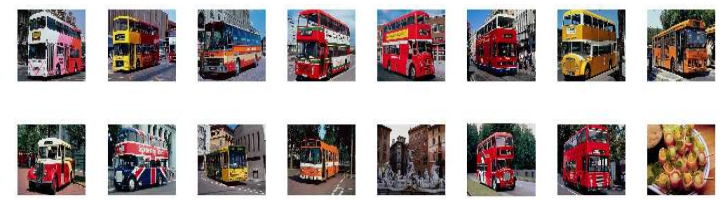

(c) The looseness texture-spatial algorithm
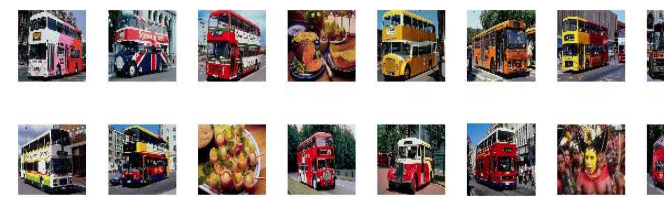

(d) MTH algorithm

Figure 6: A retrieval result of different algorithms

\section{Conclusions}

A novel retrieval algorithm based on the texture-spatial using the multiwavelets transform is presented. The improved multiwavelets quantization map is designed that depict the important visual information for all the multiwavelets sub-bands. In order to get texture-spatial feature, spatial histogram index is exacted as the texture spatial feature the multiwavelets quantization map. At the same time, the LBP model is used to describe the local texture-spatial feature for the low frequency sub-band of the multiwavelets transform. The proposed algorithm possesses not only the global spatial texture information but also the local spatial information. Experiments indicate that this method is more effective in the natural image retrieval than the looseness texture-spatial algorithm and MTH algorithm discussed in the paper. Further research could be carried out on extending the proposed method to other pattern recognition applications.

\section{Acknowledgement}

This work was supported by the National Natural Science Foundation of China (NSFC) under Grant (61173173, 61272430); Shandong province Natural Science Foundation (ZR2011FM035). Yantai science and technology development plan (2011055).

\section{References}

[1] Ju Han, Kai-Kuang Ma, Rotation-invariant and scaleinvariant Gabor features for texture image retrieval, Image and Vision Computing, Mag , 25, 1474-1481 (2007).

[2] G.Quellec, M.Lamard, G.Cazuguel, Wavelet optimization for content-based image retrieval in medical databases,Medical Image Analysis, Mag, 14, 227-241 (2010).

[3] Manesh Kokare, Biswas. Texture Image Retrieval Using New Rotated Complex Wavelet Filters, IEEE Transactions on Systems, Man, and CyberneticsłPart B: Cybernetics, Mag, 35, 1168-1178 (2005).

[4] Shang Zhaowei, Liu Guizhong, Zhao Ping. Multiwavelet Based Histogram for Texture Image Retrieval. Journal of Xian Jiaotong University, Mag, 39, 123-125 (2005).

[5] An Zhiyong. Image Retrieval Based on the Texture and Spatial Features Using the Integrated Multiwavelets Quantization Map. Journal of Computational Information Systems, Mag, 13, 5553-5560 (2012).

[6] Guang-Hai Liu. Lei Zhang, Ying-Kun Hou. Image retrieval based on multi-texton histogram. Pattern Recognition, Mag, 43, 2380-2389 (2010).

[7] C.Mariantonia, Laura B.M, Luigia P, Multiwavelets Analysis and Signal Processing, IEEE Transactions on circuits and systems, Mag, 45, 970-986 (1998).

[8] V. Strela. Multiwavelets: Theory and Application, Ph D Thesis, MIT, (1996).

[9] P. Moulin, J. Liu, Multiresolution Gray-Scale and Rotation Invariant Texture Classification with Local Binary Patterns, IEEE Transactions on Pattern Analysis and Machine Intelligence, Mag, 24, 971-987 (2002).

[10] Ch. Srinivasa rao, S. Srinivas kumar, B. N. Chatterji., Content based image retrieval using contourlet transform, ICGST-GVIP Journal, Mag, 7, 9-15 (2007). 


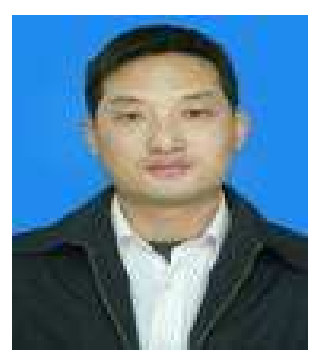

Zhiyong An received the MS degree in computer science from XiDian University in 2004, and the $\mathrm{PhD}$ degree in computer science from XiDian University in 2008. He is currently a associate professor in Shandong Institute of Business and Technology. His research interests are in the areas of image retrieval and information processing.

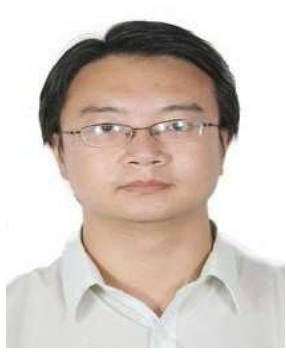

Jinjiang Li received the master's degree in applied computer from TaiYuan University of technology in 2004. From 2004 to 2006, he was a assistant engineer at institute of computer science and technology of Peking University. He awarded a Ph.D. degree in computer from Shandong University in 2010, and now work at Shandong Institute of Economic and Techonlogy. His research interests include computer graphics, computer-aided geometry design and digital image processing.

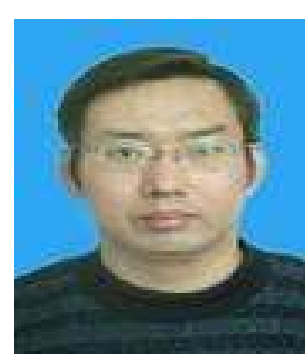

Feng Zhao received the MS degree in computer science from XiDian University in 2004, and the $\mathrm{PhD}$ degree in computer science from XiDian University in 2008. He is currently a associate professor in Shandong Institute of Business and Technology. His research interests are in the areas of patter recognition and information processing.

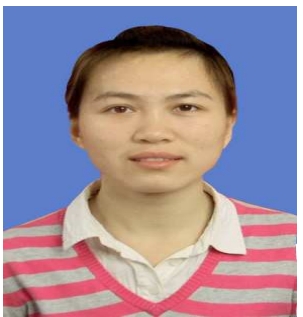

Jie Guo born in 1990. Now she is studing for her M.S.degree in computer science at Shandong Normal University. Her research interests is image processing. 